\title{
The Theory of Brain Cell Activation
}

\author{
Zuodong Sun \\ Ya'ou Brain Science Institute of Heilongjiang Province, Harbin, Heilongjiang 150090, China
}

\begin{abstract}
This is a new idea that based on effective treatment of Parkinson's disease and Alzheimer's disease with transcranial magnetoelectric stimulation technology. It is a hypothesis that Voltage gated $\mathrm{Ca}^{2+}$ channels can activate the best target by Physical means. Basic content: neurodegenerative diseases, such as Parkinson's disease and Alzheimer's disease, are closely related to voltage-gated calcium channels. The key method of treatment is activation of neurotransmitter neurons. Voltage gated $\mathrm{Ca}^{2+}$ channels are the best target for activation by physical means. The aim is to induce calcium influx to trigger synaptic vesicles released from axon terminals and release neurotransmitters. The theory of brain cell activation sets forth the principle, method and purpose of treatment of the physical gated ion channel diseases such as Alzheimer's disease, Parkinson's disease and other neural degeneration diseases, and indicates that the attempt to treat these diseases using pharmaceutical and chemical approaches could shake our confidence in conquering the diseases, and the application of physical approaches or combined application of physical and chemical approaches in the treatment of some major encephalopathy may be our main research direction in the future.
\end{abstract}

Key words: Neuronal degeneration, physical means, transcranial magnetoelectric, voltage-gated $\mathrm{Ca}^{2+}$ channels, best target.

\section{Introduction}

Parkinson's disease (PD) and Alzheimer's disease (AD) are nervous system degenerative disease. The major pathological changes in PD are dopaminergic neuronal degeneration and death in the substantia nigra which results into striatal dopamine levels decreasing, that lead to tremors, muscle stiffness, and slow movement. The application of DA precursor levodopa to compensate for the brain to reduce DA in the clinical. It is a replacement therapy, although which can obviously improve the symptoms a few years, but there will be more serious side effects after longer-term use of the drug, even can appear "open-close" effect, continue taking levodopa can't stop the progress of PD [1]. The pathological features in $\mathrm{AD}$ are massive senile plaques (SP) in the brain (major ingredient is $A \beta$ ), neurofibrillary tangles (NFTs) formed via aggregation of microtubule-associated tau proteins, and neuron loss, which will cause atrophy of brain tissue, correlations between these characteristics are unclear. At present, there is no medicines treatment for prevent and delay

Corresponding author: Zuodong Sun, researcher, research fields: brain science, neuroscience.
AD development [2]. The more recognized pathogenesis of $\mathrm{AD}$ is as hypothesis cholinergic, namely the reduction of cholinergic neurons in the brain, lead to acetylcholine (ACh) synthesis, storage, release and decrease and produce a series of clinical manifestations, with memory and identify dysfunction as the main symptom, moreover $\mathrm{ACh}$ is a kind of important neurotransmitters in brain tissue.

The view point of the article is a new idea that based on effective treatment of $\mathrm{PD}$ and $\mathrm{AD}$ with transcranial magnetoelectric (TME) stimulation technology. It is a hypothesis that Voltage gated $\mathrm{Ca}^{2+}$ channels can activate the best target by Physical means. It suggests that the basis of exocytosis maintains basic metabolic processes of degenerative dopaminergic neurons, cholinergic neurons before death, and there is a reversible process between degenerative and normal neurons, which can be used with physical means (TME) for activating dopaminergic neurons, cholinergic neurons and so on, and the voltage-gated $\mathrm{Ca}^{2+}$ channels is the best target for physical means (TME). This view supports hypothesis vesicles and hypothesis cholinergic [3]. The author made bold to name this viewpoint as "theory of brain cell activation", which is 
applicable to encephalopathy but not limited in encephalopathy.

This article is only aimed at degenerative dopaminergic neurons, cholinergic neurons, not including upstream events which may cause neuronal degeneration and downstream events which are leded to the degeneration of neurons, such as: $A \beta$, tau protein, etc.

Ion channels on the cell membrane are particular integrin which have ion selectivity and gating characteristics. The process of ion channels opening and closing its gate is called gating. Currently, the gated ion channels are subdivided into chemical, voltage-gated, and mechanical gating, etc [4]. Because sound, light, electricity, magnetism, force and heat have physical properties, in addition to the chemical gating, the voltage, and mechanical gating are categorized as physical gating, etc. Voltage-gated $\mathrm{Ca}^{2+}$ channels belong to the physical-gated ion channels.

\section{Basic Content}

$\mathrm{PD}, \mathrm{AD}$ and other neuronal degeneration diseases, are closely related to voltage-gated calcium channels. The key method of treatment is activation of neurotransmitter neurons. Voltage gated $\mathrm{Ca}^{2+}$ channels are the best target for activation by physical means. The aim is to induce calcium influx to trigger synaptic vesicles released from axon terminals and release neurotransmitters.

\section{Validation Methods}

By means of animal study test, it is a positive correlation between TME stimulation and endogenous dopamine, acetylcholine generation, and by clinical study of TME stimulation for improvement in symptoms of $\mathrm{PD}, \mathrm{AD}$ patients.

\subsection{Animal Study}

$\mathrm{PD}$ is closely related to decrease of brain dopamine levels, which has been repeatedly confirmed $[5,6]$ in the past half century. The large number of stduies have proved that there is a positive correlation between TME stimulation and endogenous dopamine generation. The effect of transcranial magnetic stimulation on Dopamine released in hippocampus, nucleus accumbens and striatum of adult male rats was detected by microdialysis in brain. The results suggested that transcranial magnetic stimulation can significantly increased dopamine concentrations in the hippocampus, nucleus accumbens, and striatum [7]. Transcranial magnetic stimulation activates dopaminergic neurons in PD rat model. It promotes the release of dopamine from the ventral striatum [8]. At the trial, partial Parkinson's monkeys were treated fitted with stimulating electrodes. The result is that electrical stimulation can improve the symptoms of monkeys. The effective electrical stimulation can increase the striatal extracellular dopamine and its metabolites with microdialysis sampling techniques combined with HPLC method [9]. Research findings that subthalamic nucleus stimulation could increase dopamine release in rat striatal cells and activate dopaminergic neurons [10]. Someone demonstrated [11-13] that electrical stimulation could promote the release of endogenous opioid peptides in the central never system. Thus it could be inferred that electrical stimulation can promote the release of neurotransmitters. Neurotransmitters and neuropeptides are coexisted within neurons [14-16]. The role of TME is far superior to pure transcranial magnetic or transcranial electric.

\subsection{Clinical Confirmation}

Since 1994, the concept that, "activation of brain cell is a key treatment of a variety of difficult encephalopathy" was presented [17]. The author continuously repeated to verify and improve the "the theory of brain cell activation" nearly two decades from theory to practice and then the other. Under the guidance of this theory, we successfully developed encephalopathy professional equipments, such as brain function rehabilitation instrument $[18,19]$ (Suitable for cerebral apoplexy sequela, vascular dementia, and 
brain atrophy), treatment instrument for Parkinson [20-22], depression [23, 24], and Alzheimer [20, 25] and other encephalopathy therapeutic apparatus, which have got through the clinical verifications in the national clinical trial sites. It has been eligible for People's Republic of China medical Device Registration Certificate and industrialization in 1996, 2011, 2011, and 2014. Because these apparatuses are perfectly safe to use in ordinary households by the patients themselves saving the instructions from clinicians. The merit speaks for itself, and yet it contributes much to the cause of our work remaining unnoticed in the field of clinical medicine. But the truth is that, from the year of 1996 to the date of my submission, the number of encephalopathy patients benefited from it is more than 50 million and the effect of feedback is exciting. One explanation for its mechanism is that the peptide neurons, dopaminergic neurons, serotonergic neurons, and cholinergic neurons are all mainly activated accordingly.

We have applied transcranial magnetoelectric stimulation to Parkinson's disease clinically. A multicenter, randomized, double-blind, self crossover control method was used to study the clinical of PD patients who met the inclusion criteria. The results showed that transcranial magnetoelectric stimulation could significantly improve the symptoms of static tremor, rigidity and slow motion in patients with Parkinson's disease. Transcranial magnetoelectric stimulation is suitable for the treatment of mild and moderate Parkinson's disease [26]. We also have applied transcranial magnetoelectric stimulation to Alzheimer's disease clinically. A multicenter, randomized, double-blind, self crossover control method was used to study the clinical of AD patients who met the inclusion criteria. The results showed that transcranial magnetoelectric stimulation could significantly improve the symptoms of memory, cognitive function, mental state and self-care ability of daily life with AD's disease. Transcranial magnetoelectric stimulation is suitable for mild, moderate Alzheimer's disease and vascular dementia is suitable for the treatment of mild and moderate Parkinson's disease [25]. The above result was also recognized by CFDA and as a part of clinical registration basis about Parkinson therapy device and Alzheimer therapy device.

\section{Discussion}

\subsection{Cellular and Molecular Mechanisms of Degeneration of Neurons}

PD is closely related to dopaminergic neuronal degeneration and death in the substantia nigra which results into striatal dopamine levels decreasing. Tyrosine comes from food directly or indirectly will be transferred into dopa in neurons under the roles of Tyrosine hydroxylase (TH). Dopa would be transferred into dopamine by the dopa decarboxylase. Dopamine was packaged into the terminal synaptic vesicles of dopaminergic neuron axons, whereas dopamine release of vesicles is completed by quick regulation process of $\mathrm{Ca}^{2+}$-dependent vesicle exocytosis [27, 28], as shown in Fig. 1. When the concentration of the intracellular flow of $\mathrm{Ca}^{2+}$ increases to a certain extent, the release of vesicles dopamine will be triggered. $\mathrm{Ca}^{2+}$ influx is controlled by the voltage-gated $\mathrm{Ca}^{2+}$ channels open induced by the membrane depolarization. Vesicle exocytosis are divided into $\mathrm{Ca}^{2+}$-dependent rapid adjustment exocytosis and the basic state exocytosis, in which basic state exocytosis is beyond control of action potentials and $\mathrm{Ca}^{2+}[3]$. Intracellular $\mathrm{Ca}^{2+}$ comes from not only the extracellular influx of $\mathrm{Ca}^{2+}$, but also comes from the synaptic calcium depot [30]. Influx superposition of $\mathrm{Ca}^{2+}$ and basic state of $\mathrm{Ca}^{2+}$ play their roles each other. If neuron releases neurotransmitters with sustained high-frequency, and accompanied by neurotransmitter releasing into the presynaptic and $\mathrm{Ca}^{2+}$ increase, which will increase the catalytic activity of $\mathrm{TH}$, and the mRNA transcription of $\mathrm{TH}$ and its synthesis enzymes gene expressions [29]. Instead, if the dopaminergic neurons can not release dopamine timely at normal rate, degeneration neurons would occur. 


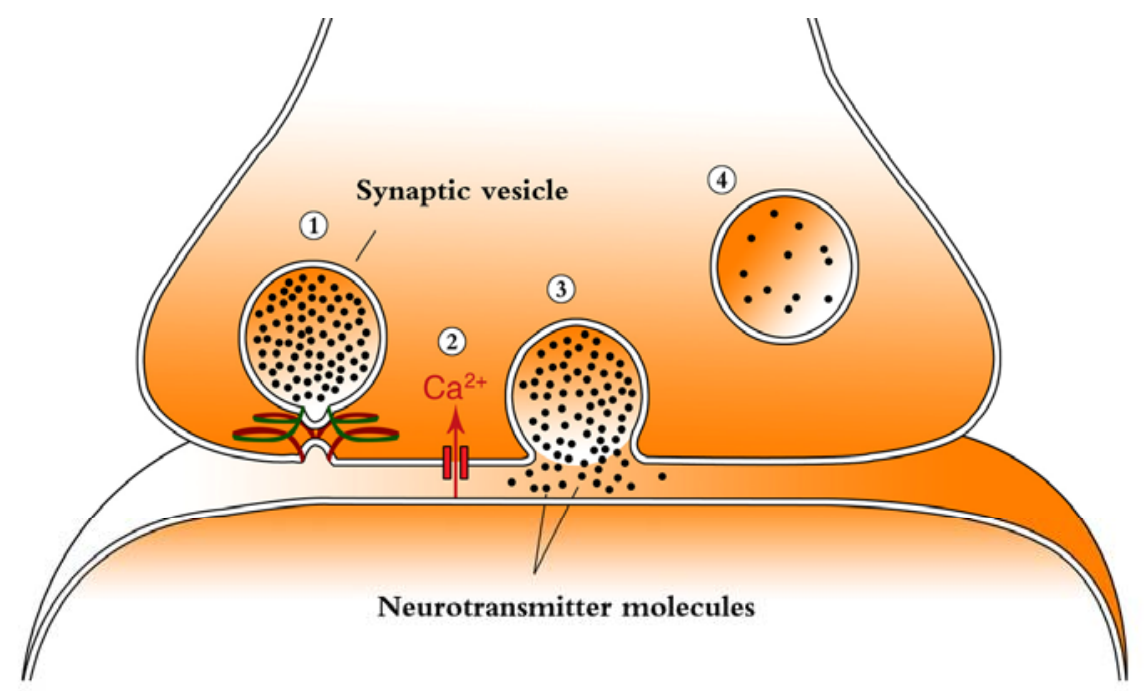

(1) Synaptic vesicles containing neurotransmitters;

(2) $\mathrm{Ca}^{2+}$ through voltage-gated channels triggers vesicles reaction;

(3) Neurotransmitter will be released into the synaptic cleft through fusion of vesicle membrane and presynaptic membrane;

(4) Vesicle will be recycled with endocytosis process finally.

Fig. 1 Neurotransmitters are released by exocytosis [29].

While increased dopamine in the shaft plasma can inhibit the activity of $\mathrm{TH}$. The feedback regulation can lead to time-dependent inhibition of dopamine synthesis, which may be related to "open-close" effect [31] appeared in the clinical use of levodopa treatment of PD. Degenerative dopaminergic neurons will gradually lose their function and become death. The basis of state exocytosis maintains its basic metabolism before death. Exogenous dopamine, relieving the patient's symptoms at the same time, will further reduce the activity of $\mathrm{TH}$. Then the vesicle fusion rate of the basis state exocytosis, which is not controlled by action potential and $\mathrm{Ca}^{2+}$, becomes lower. This replacement therapy is equivalent to abandon rescuing the degenerative dopaminergic neurons. The regulated neuronal death signaling pathway leads to destroying the balance between survival and death signal and the apoptotic program starting [32]. This seems to be an explanation for the condition those patients with PD taking levodopa for 2-5 years would lead to serious dysfunction. This explanation supports hypothesis vesicles.

Although the pathogenesis of $\mathrm{AD}$ is complex, it is difficult to use a hypothesis fully explained, but the truth that brain cholinergic neurons occur selective degeneration, and cholinergic neurons is an important biological basis of learning and memory. Acetylcholine is generated from AcetyL-CoA, which is produced during decomposition of choline and sugar, with choline acetyltransferare (ChAT) as the catalyst. Acetylcholine is synthesized in the axoplasm for axon terminals, then through acetylcholine transporter transferred to synaptic vesicles for storage. If the cholinergic neurons can not release acetylcholine timely at normal rate, degeneration of cholinergic neurons would occur, while increased acetylcholine in the shaft plasma can inhibit the activity of ChAT. Enzyme activity decreases about $50 \%$ will effect on synthetic for acetylcholine [3]. As the same, degenerative cholinergic neurons death signaling pathway upregulates and the apoptotic program starting. Existing drugs such as cholinesterase 
inhibitors, NMDA receptor antagonist for the treatment of $\mathrm{AD}$, which be aimed at not have taken place in degeneration of cholinergic neurons, this is one explanation of these drugs, that cannot prevent or delay the progression of the disease was significantly, and the research for the treatment of $\mathrm{AD}$ drugs also have taken place in many points to the degeneration of cholinergic neurons of the upstream or downstream events. This explanation supports hypothesis cholinergic.

\subsection{The Mechanism of TME on $\mathrm{Ca}^{2+}$ Channel}

Voltage-gated calcium channels belong to oversized family members of ion channels. Among of them, $\alpha 1$ subunit of $\mathrm{Ca}^{2+}$ channel is the main functional units. Experiments show that the common characteristics of $\alpha$ subunit of voltage-gated calcium channel are as follow: S4 fragment containing positive charged amino acids, plays a leading role in the activation of membrane and potential changes; S6 fragment containing certain amino acid residues, plays a key role in the inactivation of voltage-dependent calcium channels, as shown in Fig. 2. Channel activation requires the condition that there have charged amino acids or strong bipolar ion in the phospholipid bilayer membrane electric field. These gates charge or voltage receptors movement under the effect of electric field cause the channel protein conformational change, which leads to channel activation and open [33]. As a physical factor, the magnetic field has the Lorentz force on the moving charged species, and has an impact on the permeability of ions and the cell membrane potential across the membrane, which could result in configuration changes of membrane ion channel. Experimental results suggest that the impact of moderate-intensity constant magnetic field on the ion channel may be associated with movement of ion channels related charges in the cell membrane [34]. According electrophysiological characteristics, the voltage-gated $\mathrm{Ca}^{2+}$ channels are divided into L, N, P, Q, R and T other six subtypes. Different subtypes of $\mathrm{Ca}^{2+}$ channels have different activation potential, such as activation potential of the L-type calcium channel is $-10 \mathrm{mV}$, and activation potential of T-type calcium channel is $-70 \mathrm{mV}$. In fact, when the membrane potential is near to

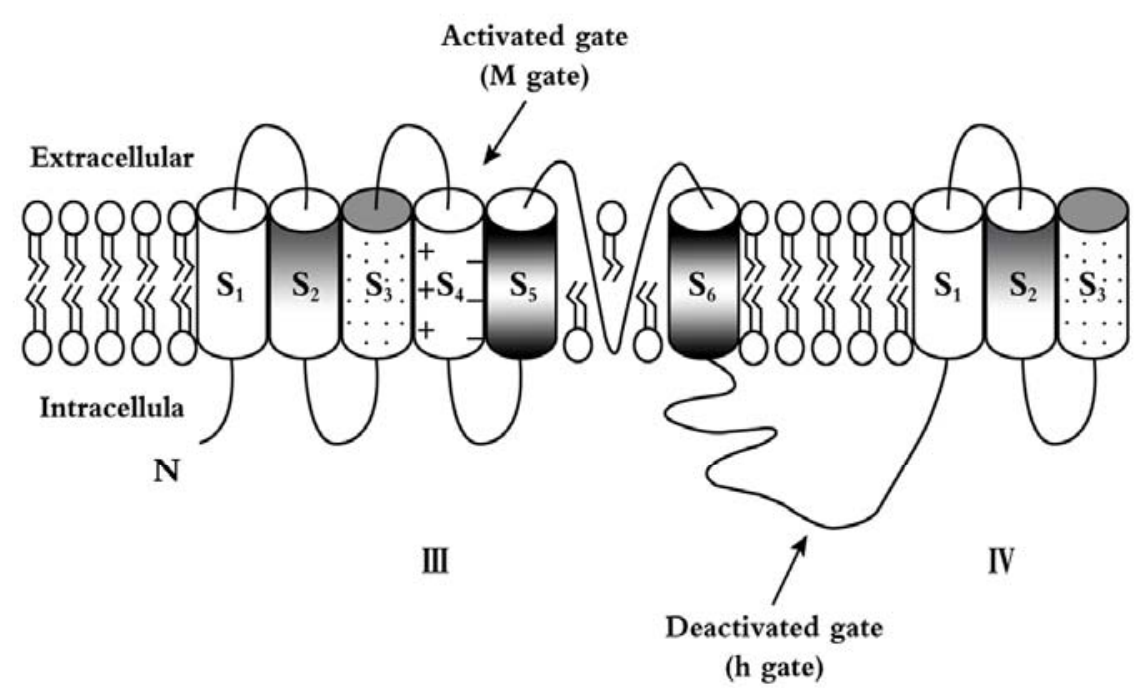

S4 is the voltage sensor, which is the activated gate (M gate). The gate will open when it is activated, and the channel is open. For some calcium channel, the peptide chains connected between III and IV are inactivation gates (h gate), the channel will be inactivated when the gate is closed.

Fig. 2 Molecular Mechanism for calcium gated channels [33]. 
$-40 \mathrm{mV}$, open probability of $\mathrm{Ca}^{2+}$ channel begins to significantly increase [3]. Under normal circumstances, the cell membrane has the intracellular calcium ion pump out of cell function, and maintain the stability of internal environment. When suffer from $A D$, this function is impaired, intracellular calcium ion overload, causing the cells inside and outside calcium ions are relatively close to the static. TME can induce $\mathrm{Ca}^{2+}$ inflowing, so will also be prompted $\mathrm{Ca}^{2+}$ outflow rapidly of the $\mathrm{Ca}^{2+}$ depot, because the stimulation is rhythmic, will cause $\mathrm{Ca}^{2+}$ concentration of two-way oscillation [37].

Electricity is the best mode of voltage gating. Transcranial magnetoelectric stimulation plays a role in the form of electricity. It activates the neurons in the major regions, activates the neurotransmitter neurons in the brain, and activates the skull with high impedance. Its safety and effectiveness are difficult to achieve by means of deep brain stimulation, high intensity transcranial magnetic stimulation or other stimuli.

\subsection{The Best Target of TME Effect}

Voltage-gated calcium channels are the best targets for physical activation, but not the only target. Voltage-gated sodium channels are the material basis for generation and propagation of action potential. While the voltage-gated calcium channels will open at the cell membrane depolarization and cause calcium influx. TME stimulation not only directly activate the calcium channels, resulting in action potential backpropagation and the sodium ion channel activation; but also can firstly activate sodium ion channels, resulting in action potential conducting along the axon to its final end and activate the calcium channels. Only activation of calcium channels can achieve the purpose that calcium influx triggers the release of vesicles neurotransmitter. Because the new technology can record the information of neurons simultaneously in multiple locations, this concept has been proved: there is back propagation in the action potential.
$\mathrm{Ca}^{2+}$ influx does not result in the uncontrolled release of neurotransmitters, even there is intense physical stimulation from the outside. There is no linear relationship between the rate of vesicle fusion and free calcium concentration [3]. This non-linear relationship makes synaptic vesicle fusion to be extremely sensitive changes for $\mathrm{Ca}^{2+}$ concentration, and which is limited to a very narrow range of $\mathrm{Ca}^{2+}$ concentration and a very short period. Regeneration of endogenous neurotransmitters including vesicle loading, transshipment and anchoring all need time and process [36, 37]. In the process of rapid vesicle exocytosis, vesicles can release to rapid depletion. The rate of vesicle release exponentially attenuated over time [3]. Neurotransmitter released into the synaptic cleft also complies with a constant internal environment doctrine. Constant internal environment is not static, but constant based on some form of rhythmic activities.

In addition, TME targets are not only limited to the voltage-gated ion channels. Such as $\mathrm{AD}$, external specific electromagnetic field is able to realize electric charge eliminating of nucleus of $\beta$-amyloid polypeptide $(A \beta)$, which is the main component of senile plaque, and deacidification of Tau protein, remove $A \beta$ or prevent $A \beta$ polymerization, and protect microtubule assembly and axone transport system.

\section{Discussions}

The views of this article is both as an deduced assertion based on the existing biochemical results, and as a new view based on the treatment with TME of PD and $\mathrm{AD}$. It is helpful for the understanding of the pathogenesis of $\mathrm{PD}$ and $\mathrm{AD}$, thus can drive and explore the safe and effective treatment of $\mathrm{PD}$ and $\mathrm{AD}$, provide a theoretical platform. T Brain cell activation theory is a theory suitable for the treatment of encephalopathy, but this theory is not limited to the treatment of encephalopathy. This theory can be used as the theoretical basis to create the "physical nosography". All the neurotransmitter-energic neuron degenerative 
diseases are closely related to voltage-gated $\mathrm{Ca}^{2+}$ channels, which can attempt to be used physical means to resolve. In fact, we also apply TME in epilepsy clinically, also has obtained the astonishing effect, while TME in pediatric cerebral palsy and awaking up persistent coma patients also has a good performance.

Brain activity is extremely complex; the authors clearly know that the new view needs more reliable supporting evidences. Either patch clamp recording or intracellular detection may cause varying degrees of damage in neurons, making it to leave the original real state. At present, we are looking for the directly authentication technology about tracking the living individual neurons with TME stimulation now. TME stimulation is related to the biological properties of membrane ion channels and channel configuration change, which need further confirmed theoretical and experimental validation of molecular biology from the molecular and cell signal transduction levels.

At present, for neuronal degeneration diseases, we seem to be more accustomed to seek a solution for the problem with drug or chemical means, and this may not be a shortcut. Blood-brain barrier (BBB) is the first barrier chemical means to be beyond. The presence of $\mathrm{BBB}$ is like defense line builded in the peripherals of central nervous system, which can not only prevent brain damage from external chemical resistance, but also keep a lot of substances being beneficial for brain outside brain [38]. Physical-gated ion channels are the second barrier chemical means to be beyond. If you want to push the door of gated ion channels occurred physical abnormality through action potentials generated by chemical means, its hardship degree may shake our confidence of coping with the diseases. The frustration on research and development of new drugs for Alzheimer's disease [39] could already prove it.

$\mathrm{PD}$ and $\mathrm{AD}$ are the worldwide difficult problems. Scientists of all countries attack for a long time with has made some research results in the chemical drug research and development in the recent hundreds of years, but is not improved substantially. Both transcranial magnetoelectric stimulation and voltage-gated calcium channels have significant physical properties. The clinical application of TME in PD and AD shows that we should pay more attention to the treatment of physical means at the same time of drug research and development. The combined application of physical therapy and chemical therapy is the research direction that we should pay more attention to in the future to conquer neuron degeneration disease thoroughly. In the treatment of patients with advanced severe PD, because of the loss of dopaminergic neurons, the combination of transcranial magnetoelectric stimulation and levodopa is a scientific treatment.

\section{References}

[1] Wang, X. J., Zhang, Y., and Chen, S. D. 2010. "Development in the Continue Decade of Pathogenesis of Parkinson's Disease and Treatment Studies." Chin. J. Contemp. Neurol. Neurosurg. 10 (1): 36-42. (in Chinese)

[2] Ge, R. 2014. "Developments Research on Alzheimer's Disease." Chinese and Foreign Medical Research 12 (9): 155-7. (in Chinese)

[3] Han, J. S. 2009. Neuro Science (The third edition). Beijing: Peking University Medical Press. (in Chinese)

[4] Hatta, S., Sakamoto, J., and Horio, Y. 2002. "Ion Channels and Diseases." Med. Electron. Microsc. 35: 117-26.

[5] Sheng, J. S. 2014. "Dysfunction of Synaptic Vesicle Recycling and Parkinson's Disease." Chemistry of Life 34 (2): 200-6. (in Chinese)

[6] Ehringer, H., and Hornykiewicz, O. 1960. "Verteilung von Noradrenalin und Dopamin (3-Hydroxytyra-min) im Gehirndes Menschen und ihr Verhalten bei Erkrankungen des Extrapyramidalen Systems.Klin.” Wochenschr 38: 1236-39. (in German)

[7] Keck, M. E., Welt, T., Müller, M. B., Erhardt, A., Ohl, F., Toschi, N., Holsboer, F., and Sillaber, I. 2002. "Repetitive Transcranial Magnetic Stimulation Increases the Release of Dopamine in the Mesolimbic and Mesostriatal System." Neuropharmacology 43: 101-9.

[8] Funamizu, H., Ogiue-ikeda, M., Mukai, H., Kawato, S., and Ueno, S. 2005. "Acuterepetitive Transcranial Magnetic Stimulation Reactivates Dopaminergic System in Lesion Rats." Neurosci. Lett. 383 (1-2): 77-81.

[9] Zhou, X. P., Liu, H. H., Gu, J., Cao, Y. Q., Li, B. M., Hu, X. W., Jiang, X. F., and Zhao, X. D. 2006. "The Changes of Neurotransmitters in the Corpus Striatum of Hemiparkinsonian Rhesus Monkeys after Deep Brain 
Stimulation of the Subthalamic Nucleus." Chin J .Neurosurg. 22 (12): 714-16. (in Chinese)

[10] Bruct, N., Windels, F., Bertrand, A., Feuerstein, C., Poupard, A., and Savasta, M. 2001. "High Frequency Stimulation of the Subthalamic Nucleus Increases the Extracellulr Contents of Striatal Dopamine in Normal and Partially Dopaminergic Denervated Rats." Neuropathol Exp Neurol 60: 15-24.

[11] Han, J. S. 2009. "Latest Progress in Research of Pain, Drug Addiction and Neurodegenerative Diseases." Journal of Peking University (Health Sciences) 41 (3): 249-54. (in Chinese)

[12] Cui, C. L., Wu, L. Z., and Luo, F. 2008. "Acupuncture for the Treatment of Drug Addiction." Neurochemical (10): 2013-22.

[13] Liang, J., Li, Y., Ping, X., Yu, P., Zuo, Y., Wu, L., Han, J. S., and Cui, C. 2006. "The Possible Involvement of Endogenous Ligands for Mu-, Delta- and Kappa-opioid Receptors in Modulating Morphine-induced CPP Expression in Rats." Peptides (27): 3307-14.

[14] Dong, X. W. 1983. "Coexistence of Neuropeptide and Proposed Neurotransmitter in Neurons." Progress in Physiological Sciences 14 (4): 328-33. (in Chinese)

[15] Hökfelt, T., Ljungdahl, A., Steinbusch, H., Verhofstad, A., Nilsson, G., Brodin, E., Pernow, B., and Goldstein, M. 1978. "Immunohistochemical Evidence of Substance P-Like Immunoreactivity in Some 5-Hydroxytryptamine-containing Neurons in the Rat Central Nervous System." Neuroscience 3: 517-38.

[16] Bigon, E., Boarato, E., Bruni, A., Leon, A., and Toffano, G. 1979. "Pharmacological Effects of Phosphatidylserine Liposomes: The Role of Lysophosphatidylserine." Br. J. Pharmac 67: 611-6.

[17] Sun, Z. D. 2003. Activate the Sleeping Brain. Harbin: Heilongjiang People's Publishing House. (in Chinese)

[18] Jiao, M. D., and Sun, Z. D. 1998. "Effects of Aobo Brain Function Rehabilitation Instrument on Cerebral Circulation and Brain Function." Medicine Healthcare Apparatus 3: 251-2. (in Chinese)

[19] Tian, N. N. 2009. "Application of Aobo Brain Function Rehabilitation Instrument in Post-troke Hemiplegia Patients." Chinese Journal of Medical Device 9: 68. (in Chinese)

[20] Sun, Z. D. 2012. Transcranial magnetoelectric encephalopathy therapeutic instrument. China Patent ZL200910071875.X, filed April 23, 2009, and issued August 22, 2012. (in Chinese)

[21] Nameless. 2011. "Successful Development of the First Therapeutic Instrument for Parkinson's Disease in China." Journal of Minimally Invasive Medicine 6 (4): 314. (in Chinese)

[22] Sun, Z. D. 2010. Conquer Parkinson. Harbin:
Heilongjiang Science \& Technology Press.

[23] Sun, Z. D. 2011. Transcranial magnetoelectric depression therapeutic instrument. China Patent ZL200910071876.4, filed April 23, 2009, and issued August 24, 2011. (in Chinese)

[24] Nameless. 2011. "Harbin Successfully Develops the First Therapeutic Instrument for Depression in the World." Science-Technology\& Publication 6: 127. (in Chinese)

[25] Nameless. 2014. "Successful Development of the First Therapeutic Instrument for Alzheimer Disease in the World." Science Technology \& Publication 6: 143. (in Chinese)

[26] Xing, X. L., and Tang, Q. 2011. "Clinical Research on Influences of Transcranial Magnetoelectric Stimulation on Parkinson's Disease." The Assembly of Conference Papers of the 11th National Rehabilitation Academic Conference of Exercise Therapy Branch of Chinese Association of Rehabilitation Medicine, Shanghai, China. (in Chinese)

[27] Hu, K., Carroll, J., Fedorovich, S., Rickman, C., Sukhodub, A., and Davletov, B. 2002. "Vesicular Restriction of Synaptobrevin Suggests a Role for Calcium in Membrane Fusion." Nature 415: 646-50.

[28] Tucker, W. C., Weber, T., and Chapman, E. R. 2004. "Reconstitution of $\mathrm{Ca}^{2+}$ Regulated Membrane Fusion by Synaptotagmin and SNAREs." Science 304 (5669): 435-8.

[29] Mark, F. B., Barry, W. C., and Michael, A. P. 2004. Neuroscience: Exploring the Brain 2th ed. Beijing: Higher Education Press.

[30] Peter, C., and Mayer, A. 1998. " $\mathrm{Ca}^{2+}$ /calmodulinsignalsthecompletion of Docking and Triggers a Late Step of Vacuole Fusion.” Nature 396: 575-50.

[31] Haavik, J., Martinea, A., and Flamark, T. 1990. "Ph-dependent Release of Catecholamined from Tyrosine Hydroxylase and Effect of Phosphorylation of Ser-40." FEBS Lett 262: 363-5.

[32] Elliott, M. R., Chekeni, F. B., Trampont, P. C., Lazarowski, E. R., Kadl, A., Walk, S. F., Park, D., Woodson, R. I., Ostankovich, M., Sharma, P., Lysiak, J. J., Harden, T. K., Leitinger, N., and Ravichandran, K. S. 2009. "Nucleotides Released by Apoptotic Cells Act as a Find-me Signal to Promote Phagocytic Clearance." Nature 461: 282-6.

[33] Yang, B. F. 2005. Ion Channel Pharmacology. BeiJing: People's medical publishing. (in Chinese)

[34] Cheng, L. J., Li, G., Lin, L., Qiao, X. Y., Zeng, R. L., Zhang, B. J., and Tong, Y. 2010. "Characteristics of Neurons Sodium Channel under Moderate Intensity Static Magnetic Field." Nanotechnology and Precision Engineering 8 (6): 559-64. (in Chinese)

[35] Zhang, S., Cui, J., and Shen, P. P. 2007. "The Regulation of Calcium Signaling in Apoptosis." Chinese Journal of Cell Biology 29: 785-90. (in Chinese) 
[36] Cabin, D. E., Shimazu, K., Murphy, D., Cole, N. B., Gottschalk, W., Mcilwain, K. L., Orrison, B., Chen, A., Ellis, C. E., Paylor, R., Lu, B., and Nussbaum, R. L. 2002. "Synaptic Vesicle Depletion Correlates with Attenuated Synaptic Responses to Prolonged Repetitive Stimulation in Mice Lacking Alphasynuclein." Neurosci 22 (20): 8797-807.

[37] Nemani, V. M., Lu, W., Berge, V., Nakamura, K., Onoa, B., Lee, M. K., Chaudhry, F. A., Nicoll, R. A., and Edwards, R. H. 2010. "Increased Expression of
Alpha-synuclein Reduces Neurotransmitter Release by Inhibiting Synaptic Vesicle Reclustering after Endocytosis." Neuron 65 (1): 66-79.

[38] Foust, K. D., Nurre, E., Montgomery, C. L., Hernandez, A., Chan, C. M., and Kaspar, B. K. 2009. "Intravascular AAV9 Preferentially Targets Neonatal Neurons and Adult Astrocytes." Nature biotechnology 27 (1): 59-65.

[39] Yin, M. 2014. "Full-scale Setback and Thinking in R\&D of Alzheimer's Disease Drug." Acta Pharmaceutica Sinica 49 (6): 757-63. (in Chinese) 